\title{
BAYESIAN MATCHEd-FieLd GeOACOUSTIC INVERSION
}

\author{
Stan E. Dosso \\ School of Earth and Ocean Sciences, University of Victoria, Victoria BC Canada V8W 3P6, sdosso@uvic.ca
}

\section{INTRODUCTION}

This paper describes a Bayesian approach to matched-field geoacoustic inversion, with emphasis on rigorous uncertainty estimation [1]-[3]. In a Bayesian formulation, the unknown geoacoustic model parameters are considered to be random variables constrained by the data and prior information, and the goal is to interpret the multidimensional posterior probability density (PPD). The approach is developed and illustrated for a shallow-water test site in the Mediterranean Sea.

\section{THEORY}

Let $\mathbf{m}$ and $\mathbf{d}$ represent the model and data vectors, respectively, with elements considered random variables that obey Bayes rule, which may be written

$$
P(\mathbf{m} \mid \mathbf{d}) \propto L(\mathbf{m}, \mathbf{d}) P(\mathbf{d}) .
$$

In the above equation, $P(\mathbf{m} \mid \mathbf{d})$ represents the PPD which quantifies the information content for the model parameters given both data information, represented by the likelihood function $L(\mathbf{m}, \mathbf{d})$, and prior information $P(\mathbf{m})$. The likelihood can typically be written $L(\mathbf{m}, \mathbf{d}) \propto \exp [-E(\mathbf{m}, \mathbf{d})]$, where $E$ represents an appropriate data misfit function (considered later). The PPD can be written

$$
P(\mathbf{m} \mid \mathbf{d})=\frac{\exp [-\phi(\mathbf{m}, \mathbf{d})]}{\int \exp \left[-\phi\left(\mathbf{m}^{\prime}, \mathbf{d}\right)\right] d \mathbf{m}^{\prime}},
$$

where the integration spans the parameter space and the generalized misfit, including data and prior, is given by

$$
\phi(\mathbf{m}, \mathbf{d})=E(\mathbf{m}, \mathbf{d})-\ln P(\mathbf{m}) .
$$

The multi-dimensional PPD is typically characterized in terms of parameter estimates, uncertainties, and interrelationships, as given by the maximum a posteriori (MAP) model, mean model, marginal probability distributions, and covariance/correlations defined

$$
\begin{aligned}
& \hat{\mathbf{m}}=\operatorname{Arg}_{\max }\{P(\mathbf{m} \mid \mathbf{d})\} \\
& \overline{\mathbf{m}}=\int \mathbf{m} P(\mathbf{m} \mid \mathbf{d}) d \mathbf{m} \\
& P\left(m_{i} \mid \mathbf{d}\right)=\int \delta\left(m_{i}-m_{i}^{\prime}\right) P\left(\mathbf{m}^{\prime} \mid \mathbf{d}\right) d \mathbf{m}^{\prime}
\end{aligned}
$$

$$
C_{i j}=\int\left(m_{i}-\bar{m}_{i}\right)\left(m_{j}-\bar{m}_{j}\right) P(\mathbf{m} \mid \mathbf{d}) d \mathbf{m} .
$$

For nonlinear problems, such as geoacoustic inversion, analytic solutions to the above optimization and integrations are not available, and numerical methods must be employed. Hybrid algorithms, such as adaptive simplex simulated annealing [1], which combine global and local methods, have proven effective for optimization. Integration can be carried out using Markov-chain Monte Carlo importance sampling methods, such as fast Gibbs sampling [2].

The data uncertainty distribution, which defines the likelihood function, must include both measurement errors (e.g., additive noise, instrument uncertainties) and theory errors (due to the simplified model parameterization and inexact theory). Since data uncertainties are generally not well known a priori, physically reasonable assumptions are required about the form of the distribution. In many practical cases, lack of specific knowledge of uncertainties suggests a simple distribution (e.g., Gaussian) be assumed with statistical quantities estimated from the data.

Let the complex acoustic pressure fields measured at an array of $N$ sensors and $F$ frequencies be given by $\mathbf{d}=\left\{\mathbf{d}_{f}\right.$, $f=1, F\}$. Assuming the data errors are complex, circularlysymmetric, zero-mean Gaussian-distributed random variables which are uncorrelated from frequency to frequency but potentially correlated spatially with data covariance matrix $\mathbf{C}_{f}$ at the $f$ th frequency, the likelihood function is given by $L(\mathbf{m}, \mathbf{d}) \propto \prod_{f=1}^{F} \exp \left\{\left[\mathbf{d}-A_{j} e^{i \theta_{j}} \mathbf{d}_{f}(\mathbf{m})\right]^{T} \mathbf{C}_{j}^{-1}\left[\mathbf{d}-A_{j} e^{i \theta_{j}} d_{j}(\mathbf{m})\right]\right\}$ where $T$ indicates conjugate transpose, $\mathbf{d} /(\mathbf{m})$ is the modeled acoustic pressure, and $A_{f}$ and $\theta_{f}$ represent the unknown source spectrum. Maximizing the likelihood with respect to $A_{f}$ and $\theta_{f}$ leads to

$$
L(\mathbf{m}, \mathbf{d}) \propto \exp \left\{-\sum_{f=1}^{F} \mathbf{d}_{j}^{T} \mathbf{C}_{f}^{-1} \mathbf{d}_{j}+\frac{\left|\mathbf{d}_{f}^{T} \mathbf{C}_{f}^{-1} \mathbf{d}_{f}(\mathbf{m})\right|^{2}}{\left|\mathbf{d}_{f}^{T}(\mathbf{m}) \mathbf{C}_{f}^{-1} \mathbf{d}_{f}(\mathbf{m})\right|}\right\} .
$$

The data covariance matrices $\mathbf{C}_{f}$ are generally not known $a$ priori, but can be estimated from the autocovariance of the data residuals (difference between measured data and data computed for the MAP model estimate, applying maximumlikelihood source spectral estimates), under the assumption of ergodicity [3]. The validity of the covariance estimates and the assumption of Gaussian error processes can be examined by applying statistical tests to the data residuals, 
standardized by the Cholesky decomposition (square root) of the inverse covariance matrix. For instance, the runs test can be applied to examine whether the estimated covariance matrices successfully decorrelates the residuals, and the Kolmogorov-Smirnov (KS) test can be applied to examine the Gaussianity of the residuals [3].

\section{RESULTS}

The geoacoustic experiment was carried out by the NATO Undersea Research Centre in the Mediterranean Sea off the west coast of Italy near Elba Island. The experiment consisted of recording acoustic signals from a transducer towed at approximately $12-\mathrm{m}$ depth over a track with nearly range-independent bathymetry (water depth $\sim 132 \mathrm{~m}$ ). The source emitted a 0.5 -s linear frequency-modulated signal over the band $300-800 \mathrm{~Hz}$ every $0.25 \mathrm{~km}$. The signals were received at a bottom-moored vertical line array (VLA) of 48 hydrophones which spanned from 26-120-m depth with 2-m sensor spacing. The data set analyzed here consisted of 11 frequencies at $50-\mathrm{Hz}$ intervals from $300-800 \mathrm{~Hz}$ recorded for a source-receiver range of approximately $3.85 \mathrm{~km}$.

The experiment and the environmental and geometric parameters included in the model $\mathbf{m}$ are illustrated in Figure 1. The acoustic source is at depth $z$ and range $r$ from the VLA in water of depth $D$. The geoacoustic parameters include the thickness $h$ of an upper sediment layer with sound speed $c_{s}$, density $\rho_{s}$, and attenuation $\alpha_{s}$, overlying a semi-infinite basement with sound speed $c_{b}$, density $\rho_{b}$ and attenuation $\alpha_{b}$. The SSP is represented by four unknown sound speeds $c_{1}-c_{4}$ at depths of $0,10,50$, and $D \mathrm{~m}$.

The Bayesian inversion applied bounded uniform prior distributions for all parameters. Wide bounds were applied for the geoacoustic parameters to limit the inversion to physically reasonable values but allow the acoustic data to determine the solution. For the SSP parameters, the bounds were $\pm 4 \mathrm{~m} / \mathrm{s}$ about the measured sound speeds, representing calibration uncertainty and the effects of spatial and/or temporal variability. For the geometric parameters $(D, r, z)$, the bounds represent small corrections due to imprecise knowledge of these experiment parameters.

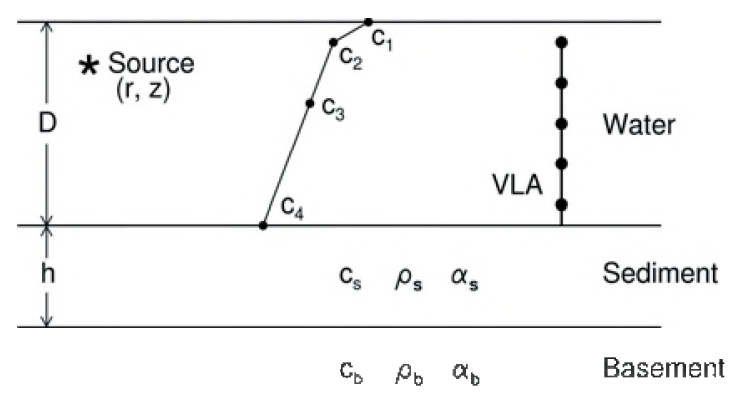

Fig. 1. Experiment geometry and model parameters.

Due to space limitations, inversion results are shown here only in terms of parameter marginal distributions (Figure 2). With the exception of the basement density, the geoacoustic parameters are generally well determined, with the sediment thickness and basement sound speed particularly well resolved. The SSP parameters are less well determined, due to strong inter-parameter correlations (not shown). The geometric parameters are distributed near their nominal values. The KS test indicated no significant evidence against the assumption of Gaussian-distributed error processes, while the runs test suggested that the estimated data covariance matrices accounted for much, but not all, of the data error correlations.

\section{REFERENCES}

[1] Dosso, S. E., M. J. Wilmut and A. L. Lapinski (2001). An adaptive hybrid algorithm for geoacoustic inversion. IEEE. J. Ocean. Eng.., vol 26, 324-336.

[2] Dosso, S. E. and P. L. Nielsen (2002). Quantifying uncertainty in geoacoustic inversion II: A fast Gibbs sampler approach. J. Acoust. Soc. Am., vol 111, 143-159.

[3] Dosso, S. E., P. L. Nielsen and M. J. Wilmut (2006). Data error covariances in matched-field geoacoustic inversion. J. Acoust. Soc. Am., vol 119, 208-219.

\section{ACKNOWLEDGEMENTS}

The author thanks Peter Nielsen of the NATO Undersea Research Centre for providing the acoustic data and for helpful discussions.
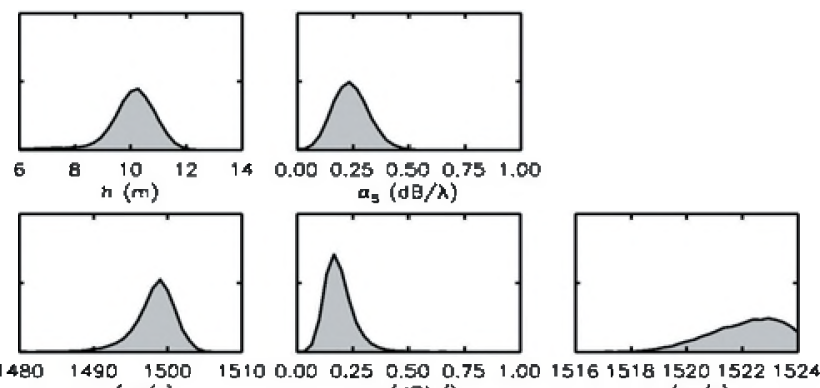
$c_{\mathrm{s}}(\mathrm{m} / \mathrm{s})$ $a_{B}(\mathrm{de} \lambda /)$ $\mathrm{s},(\mathrm{m} / \mathrm{s})$
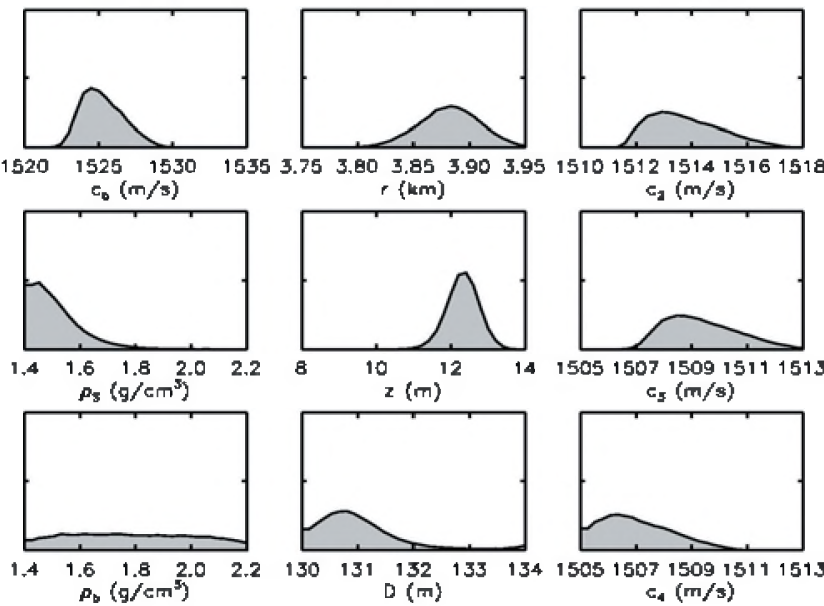

, $(\mathrm{km}$ $G_{1}(\mathrm{~m} / \mathrm{s})$
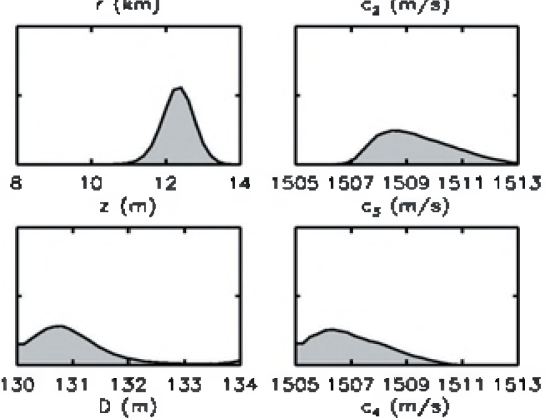

Fig. 2. Posterior Marginal probability distributions. 